\title{
Interchromosomal template-switching as a novel molecular mechanism for imprinting perturbations associated with Temple syndrome
}

Claudia M. B. Carvalho ${ }^{1 *}$, Zeynep Coban-Akdemir ${ }^{1}$, Hadia Hijazi ${ }^{1}$, Bo Yuan ${ }^{1}$, Matthew Pendleton², Eoghan Harrington ${ }^{2}$, John Beaulaurier ${ }^{3}$, Sissel Juul ${ }^{2,3}$, Daniel J. Turner ${ }^{4}$, Rupa S. Kanchi ${ }^{5}$, Shalini N. Jhangiani ${ }^{6}$, Donna M. Muzny ${ }^{6}$, Richard A. Gibbs ${ }^{1,6}$, Baylor-Hopkins Center for Mendelian Genomics, Pawel Stankiewicz', John W. Belmont ${ }^{1,7}$, Chad A. Shaw ${ }^{1}$, Sau Wai Cheung ${ }^{1}$, Neil A. Hanchard', V. Reid Sutton ${ }^{1,8}$, Patricia I. Bader ${ }^{9}$ and James R. Lupski ${ }^{1,6,7,8}$

\begin{abstract}
Background: Intrachromosomal triplications (TRP) can contribute to disease etiology via gene dosage effects, gene disruption, position effects, or fusion gene formation. Recently, post-zygotic de novo triplications adjacent to copynumber neutral genomic intervals with runs of homozygosity $(\mathrm{ROH})$ have been shown to result in uniparental isodisomy (UPD). The genomic structure of these complex genomic rearrangements (CGRs) shows a consistent pattern of an inverted triplication flanked by duplications (DUP-TRP/INV-DUP) formed by an iterative DNA replisome template-switching mechanism during replicative repair of a single-ended, double-stranded DNA (seDNA), the ROH results from an interhomolog or nonsister chromatid template switch. It has been postulated that these CGRs may lead to genetic abnormalities in carriers due to dosage-sensitive genes mapping within the copy-number variant regions, homozygosity for alleles at a locus causing an autosomal recessive (AR) disease trait within the ROH region, or imprinting-associated diseases.
\end{abstract}

Methods: Here, we report a family wherein the affected subject carries a de novo 2.2-Mb TRP followed by $42.2 \mathrm{Mb}$ of $\mathrm{ROH}$ and manifests clinical features overlapping with those observed in association with chromosome 14 maternal UPD (UPD(14)mat). UPD(14)mat can cause clinical phenotypic features enabling a diagnosis of Temple syndrome. This CGR was then molecularly characterized by high-density custom aCGH, genome-wide single-nucleotide polymorphism (SNP) and methylation arrays, exome sequencing (ES), and the Oxford Nanopore long-read sequencing technology.

Results: We confirmed the postulated DUP-TRP/INV-DUP structure by multiple orthogonal genomic technologies in the proband. The methylation status of known differentially methylated regions (DMRs) on chromosome 14 revealed that the subject shows the typical methylation pattern of UPD(14)mat. Consistent with these molecular findings, the clinical features overlap with those observed in Temple syndrome, including speech delay.

(Continued on next page)

\footnotetext{
* Correspondence: cfonseca@bcm.edu

'Department of Molecular and Human Genetics, Baylor College of Medicine,

One Baylor Plaza, Room 604B, Houston, TX 77030-3498, USA

Full list of author information is available at the end of the article
}

(c) The Author(s). 2019 Open Access This article is distributed under the terms of the Creative Commons Attribution 4.0 International License (http://creativecommons.org/licenses/by/4.0/), which permits unrestricted use, distribution, and reproduction in any medium, provided you give appropriate credit to the original author(s) and the source, provide a link to the Creative Commons license, and indicate if changes were made. The Creative Commons Public Domain Dedication waiver (http://creativecommons.org/publicdomain/zero/1.0/) applies to the data made available in this article, unless otherwise stated. 
(Continued from previous page)

Conclusions: These data provide experimental evidence that, in humans, triplication can lead to segmental UPD and imprinting disease. Importantly, genotype/phenotype analyses further reveal how a post-zygotically generated complex structural variant, resulting from a replication-based mutational mechanism, contributes to expanding the clinical phenotype of known genetic syndromes. Mechanistically, such events can distort transmission genetics resulting in homozygosity at a locus for which only one parent is a carrier as well as cause imprinting diseases.

Keywords: Triplication, DUP-TRP/INV-DUP, Complex genomic rearrangement, MMBIR, Replicative-based mechanism, Inter-homologous chromosomal template switch, Runs of homozygosity (ROH), ES, Absence of heterozygosity $(\mathrm{AOH})$

\section{Background}

Intrachromosomal triplications (TRP) are defined as a heterozygous segmental 3X copy-number amplification of a given locus in which all three copies are inserted in cis. For a TRP locus, the total number of alleles will be equivalent to four copies for autosomes and equal to three copies for the X-chromosome of 46, XY males [1]. TRP can potentially contribute to disease etiology via gene dosage effects $[2,3]$, gene disruption at breakpoint junctions [4], position effects [5, 6], or fusion gene formation [7]. Triplication of dosage-sensitive genes can increase phenotypic severity in triplication versus duplication carriers [2, 3, 8-11], lower the disease age-of-onset [12], or result in distinct clinical phenotypes [13] compared to duplications affecting the same locus. Moreover, locus duplication increases the risk of triplication formation as a result of unequal crossover (i.e., nonallelic homologous recombination; NAHR) by about 100X [11, 14, 15]; such type of rearrangement within a given locus can result in altered inter-generational disease penetrance [16-18] with the appearance of genetic anticipation [11].

The contribution and impact of triplication on disease etiology is still under-recognized [19] due, in part, to the technical limitations in distinguishing duplications from triplications or higher order (e.g. quadruplication and further amplification) copy-number gains [11, 18]; this molecular diagnostic challenge may result in triplications being miscalled as duplications. We and others have reported a rare type of de novo complex genomic rearrangement (CGR) constituted by TRP followed by absence of heterozygosity $(\mathrm{AOH})$ in patients clinically referred for multiple congenital abnormalities [20-24]. TRP segments are inverted and can be as large as $21.7 \mathrm{Mb}$ in size in addition to being flanked by small duplications of a few $\mathrm{Kb}$, a structure named inverted triplication flanked by duplications (DUP-TRP/INV-DUP) [10, 22]. The copy-number neutral $\mathrm{AOH}$ segment can encompass $>50 \mathrm{Mb}$ extending to the telomere and reflects segmental uniparental isodisomy (UPD) whereas the triplications are formed by biparental contributions [22], and both variant types are potentially generated concomitantly in a post-zygotic event [22]. Genotype-phenotype correlations suggest that clinical abnormalities result from gene dosage and expression alterations of genes included within the copy-number gain interval(s) [24] and from unmasking of biallelic autosomal recessive (AR) disease traits; as an example of the latter, Sahoo and colleagues reported a patient with autosomal recessive citrullinemia (MIM\#215700) in whom a paternal UPD segment included a paternally inherited pathogenic variant in ASS1 [23]. Moreover, some of the subjects with $\mathrm{TRP} / \mathrm{AOH}$ may also present clinical phenotypes due to the inheritance of an imprinted locus within the segmental UPD, which was predicted based on the underlying formation mechanism but not yet reported in patients with genomic disorders [25].

Here, we report an individual proband and family in which family-based genomic analyses revealed a de novo 2.2-Mb TRP followed by $42.2 \mathrm{Mb}$ of $\mathrm{AOH}$ involving chromosome 14 in the proband. Segregation analysis of single-nucleotide polymorphism (SNP) array data indicates that the copy-number neutral runs of homozygosity $(\mathrm{ROH})$ segment results from maternal uniparental isodisomy for chromosome 14 (UPD(14)mat) [26], a molecular diagnosis which may lead to a clinical diagnosis of Temple syndrome [MIM\#616222]. Further assessment of differentially methylated regions (DMR) within the DLK1-DIO3 locus, using independent genomic approaches of genome-wide methylation arrays and nanopore sequencing, confirmed the typical methylation pattern observed in UPD(14)mat patients; the index case indeed presents clinical features overlapping those observed with Temple syndrome. In conclusion, our data provide experimental evidence that, in humans, post-zygotically generated in cis triplications can also present with segmental UPD in association with altered methylation and imprinting-associated disease due to the loss of one parental contribution at a locus. This further increases the potential genomic and pathogenetic consequences of this type of structural variant mutagenesis event and resultant CGR.

\section{Methods}

\section{Subjects}

Index subject, BAB7004, carrying the triplication associated with $\mathrm{AOH}$ was identified by the Baylor Genetics clinical diagnostic laboratory by chromosome microarray analysis (CMA) [27, 28] as part of their clinical 
evaluation. Informed consent was obtained for participation in further genome-wide research studies of all family members, protocols no. $\mathrm{H}-25466$ and/or no. H-29697, both approved by the institutional review board at Baylor College of Medicine. Genomic DNA from the index patient, BAB7004, and unaffected family members (family pedigree HOU2583): BAB7088 (father), BAB7291 (mother), and siblings, BAB7085, BAB7086, BAB7087, and BAB7292, was isolated from blood according to standard procedures. For the genome-wide methylation assay controls, we used samples from subjects previously diagnosed as carriers of maternal uniparental disomy 14 (UPD(14)mat): BAB489 [26], BAB7704, BAB7705, and paternal uniparental disomy 14 (UPD(14)pat): BAB7706 [29]. DNA was extracted from either blood (BAB489) or lymphoblastoid cell lines (BAB7704, BAB7705, and BAB7706).

\section{Determining triplication size and genomic intervals of absence of heterozygosity (AOH)}

Structural variation and $\mathrm{AOH}$ in subject BAB7004 were identified by clinical chromosome microarray analysis (CMA) using V10.1 OLIGO [28] which is a custom-designed array with approximately 400,000 interrogating oligonucleotides that include 60,000 probes used for assaying single-nucleotide polymorphisms (SNPs) (Agilent Technologies, Inc., Santa Clara, CA, USA) [28]. To confirm the presence of the $\mathrm{AOH}$ segment, as well as to access the origin of triplicated genomic segments, samples were further submitted for SNP array analysis. DNA samples from all family members. Subjects BAB7004, BAB7088, BAB7291, BAB7085, BAB7086, BAB7087, and BAB7292 were hybridized on a HumanOmniExpress-24 Beadchip at the Human Genome Sequencing Center of Baylor College of Medicine. Basic quality control and analysis of the genotyping data were performed using GenomeStudio software (Illumina, Inc., San Diego, CA, USA). $\mathrm{B}$-allele frequency, $\mathrm{BAF}$ is calculated as: $B /(A+B)$, where $\mathrm{A}$ is the signal of the A allele and $\mathrm{B}$ is the signal of the $\mathrm{B}$ allele. We further identified $\mathrm{AOH}$ segments from unphased exome-sequencing (ES) data using BAM files and preprocessing VCFs according to the BafCalculator algorithm described previously $[30,31]$.

\section{Refining triplication size and gene content}

To refine the breakpoint junctions of the triplication, we designed a high-density custom tiling-path oligonucleotide microarray spanning chromosome $14 \mathrm{q}$ using the Agilent SureDesign website (https://earray.chem.agilent.com/suredesign/website). The average coverage was 1 probe per $1000 \mathrm{bp}$, spanning the following genomic interval (hg19) chr14: $80,000,000-105,000,000$ as described [22]. Probe labeling and hybridization were performed according to the manufacturer's protocols with minor modifications.

\section{Oxford Nanopore long read sequencing}

\section{Sample preparation, sequencing, and base-calling}

For this patient sample, two parallel libraries were generated, one using sheared DNA and one using "full-length" DNA. Both libraries were generated from 5- $\mu$ g genomic DNA isolated from blood. The sheared library was generated by centrifugation in a g-TUBE (Covaris part number 520079) at 1086 rcf. Library preparation was performed in parallel for both sheared and unsheared samples using Oxford Nanopore Technologies' standard ligation sequencing kit, SQK-LSK109, using $1 \mu \mathrm{g}$ of the appropriate genomic DNA (gDNA) and the standard protocol with a slight modification in that the final elution was done in $24 \mu \mathrm{l}$ elution buffer instead of the standard $12 \mu \mathrm{l}$. The $24 \mu \mathrm{l}$ of library was combined with $75 \mu \mathrm{l}$ sequencing buffer and $51 \mu \mathrm{l}$ of loading beads and run on a PromethION Beta with PromethION flow cell version 3 using MinKNOW software version 1.11.9 for $64 \mathrm{~h}$. Base-calling was performed using Guppy version 1.4 .0 and using a manually set chunk size of $10 \mathrm{~kb}$.

\section{Analysis of long-read sequencing data}

Alignment of the reads for samples was performed with ngmlr (version 0.2.7) [32] using default parameters along with the -bam-fix parameter for long cigar string support. Structural variant calling was performed with the Sniffles structural variant caller (version 1.0.8) [32], using the following parameter set, chosen to maximize sensitivity “--min support 1 --minmapping qual 1 --num reads_report -1 --genotype". Suspected structural variants (SV) were confirmed as a concordance between changes in the read depth and the breakpoints for SV events. Upon identification of suspected causal rearrangements, the read names supporting the putative variant were pulled from the sniffles vcf file, and the full collection of mappings for that read were pulled from the bam file, in order to confirm the breakpoint manually in IGV [33], followed by PCR and Sanger sequencing. Sequencing depth was calculated on the resulting alignments using mosdepth (version 0.2.3) [34] with the following parameter set "-F 3588 -Q 1" which calculates coverage depth in $100 \mathrm{bp}$ bins and includes only primary and supplemental alignments. Sniffles and mosdepth were run on the pooled combination of both sheared and unsheared library preparation strategies to maximize the probability of getting reads that unambiguously resolve the breakpoint junctions. Read depth coverage in $100 \mathrm{bp}$ bins was plotted using $\mathrm{R}$ ggplot2 package in this region among relative copy-number states (normal, triplication, and duplication regions). The median value of read depth coverage was reported for each copy-number state within the breakpoint junctions that had been mapped to the nucleotide level. 


\section{Methylation analysis}

Nanopore reads were first aligned to GRCh37 using minimap2 v2.15 [35] using the options "-ax map-ont". Read alignments overlapping MEG3-DMR (chr14: 101,290,524$101,293,978$ ) were extracted, and the reads were assigned to haplotypes using marginPhase v1.0.0 with the supplied model "params.nanopore.json". For reads from each haplotype assignment, methylation status of $\mathrm{CpG}$ sites was assessed using nanopolish call-methylation [36]. Log-likelihood ratios were produced for $\mathrm{CpG}$ sites on each read, where positive and negative values indicate likely methylation and nonmethylation, respectively.

\section{Long-range PCR amplification and breakpoint junction validation}

Reverse and forward primer pairs (relative to the reference genome) were designed at the apparent boundaries of the copy-number gain segments as defined by high-density aCGH analysis for triplications and by mapping of the transition interval from copy-number neutral to copy-number gain positions [10]. Long-range PCR was performed using TaKaRa LA Taq (Clontech, Mountain View, CA, USA). PCR sample-specific products were sequenced by the Sanger di-deoxy sequencing methodology. PCR and sequencing results were independently repeated to confirm results. Parental DNA was amplified in the same experiments, i.e., used as a negative control and to determine that the breakpoint junction specific PCR was exclusively present in the index subject (i.e., a de novo event). Junction 1 primers are as follows: 7004-trp1F, GGACAGGTAGAGA GCACCCAGTTCC; 7004-dup1F, ACAGCCCCACCACC TTATTTTGAAG. Junction 2 primers: 7004_TRP2NR: TG AAATCAGTGAGTCATGGCTG; 7004_DUP2NR: AAAT CAATGAGACTTGGCTGTTC.

\section{Exome sequencing (ES), variant calling, and selection of de novo and biallelic variants specifying AR disease trait loci affecting chromosome 14}

ES was performed at the Human Genome Sequencing Center (HGSC) at Baylor College of Medicine through the Baylor-Hopkins Center for Mendelian Genomics initiative. Using $0.5 \mu \mathrm{g}$ of DNA an Illumina paired-end pre-capture library was constructed according to the manufacturer's protocol (Illumina Multiplexing_SamplePrep_Guide_1005361_D) with modifications as described in the BCM-HGSC protocol (https://www.hgsc. $\mathrm{bcm}$.edu/content/protocols-sequencing-library-construction). Six pre-captured libraries were pooled and then hybridized in solution to the HGSC VCRome 2.1 design [37] (42 Mb NimbleGen, Cat. No. 06266380001) according to the manufacturer's protocol NimbleGen SeqCap EZ Exome Library SR User's Guide with minor revisions. The sequencing run was performed in paired-end mode using the Illumina HiSeq 2000 platform, with sequencing-by-synthesis reactions extended for 101 cycles from each end and an additional seven cycles for the index read. With a sequencing yield of 6.6 Gb, the samples achieved $95 \%$ of the targeted exome bases covered to a depth of $20 \mathrm{X}$ or greater. Illumina sequence analysis was performed using the HGSC Mercury analysis pipeline [38, 39] (https://www.hgsc.bcm.edu/software/mercury) which moves data through various analysis tools from the initial sequence generation on the instrument to annotated variant calls (SNVs and intra-read in/dels). De novo variants were identified in silico by subtracting variants observed in either parent (DNM-Finder; https://github.com/BCM-Lupskilab/DNM-Finder) [40]. Candidate variants/mutations were filtered against publicly available databases including the 1000 Genomes Project, NHBLI GO Exome Sequencing Project (ESP), the Atherosclerosis Risk in Communities Study (ARIC) database, and our in-house generated database of greater than 11,000 exomes. Recessive disease trait specifying biallelic variants affecting disease-associated genes mapping to human chromosome 14 was selected for Sanger di-deoxy DNA sequencing confirmation and to evaluate the transmission genetics segregation pattern in affected and unaffected family members. Prediction scores from four distinct computational algorithms were used to assess pathogenicity of rare variants: PolyPhen-2 [41], SIFT [42], LRT [43] and MutationTaster [44], combined annotation-dependent depletion (CADD) [45]. Copy-number variants (CNVs) using ES were called using XHMM [46].

\section{Variant confirmation}

Potential disease-associated variants identified via ES were verified and evaluated for co-segregation with the clinically observed phenotype using standard PCR amplification. PCR products were purified using ExoSAP-IT (Affymetrix) and sequenced using di-deoxy nucleotide Sanger sequencing at the DNA sequencing core at BCM.

\section{Chromosome 14 methylation profiling}

The specific chromosome 14 methylation profile was determined by analyzing the methylation profile across $\mathrm{CpG}$ sites included in the Infinium Human Methylation450 Beadchip (Illumina). DNA samples studied included all family HOU2583 members (affected and unaffected family members) for family-based genomic analysis, samples previously known to carry UPD(14)mat or UPD(14)pat were used as controls. Data analysis was performed using the methylation module of the GenomeStudio Software 2011.1 (Illumina).

DNA samples were first subject to bisulphite conversion using the EZ-96 DNA Methylation kit (Zymo Research Corp., Irvine, California, USA). Bisulfite converted DNA was then profiled using the Infinium 
HumanMethylation450 Beadarray (Illumina) according to the manufacturer's instructions. The resulting genome-wide array intensity files (iDat) were imported into the methylation module (version 1.9.0) of GenomeStudio (v2011.1, Illumina) with background normalization and used to generate methylation beta-values for each of the $\sim 450,000$ loci/sites interrogated on the array. All individuals had less than $5 \%$ missing data across all probes. Probes with a detection $p$ value $>0.0001$ were removed. Our primary experimental goal with this assay was to observe large differences in methylation on chromosome 14 corresponding to imprinted sites; therefore, we did not employ further normalization for color balance or channel, or tissue deconvolution of samples prior to analysis. Probes assigned to chromosome 14 on the accompanying $450 \mathrm{~K}$ manifest were filtered to remove low-intensity probes, exported as a data table to RStudio ( $\mathrm{v} 1.0 .143$ ), and analyzed using R (v3.1.3). Downstream analyses involved assigning the resulting $\beta$ values (i.e., proportion of methylated cytosines) at a given $\mathrm{CpG}$ probe site as follows: $\beta>0.8$ methylated, $0.2>\beta<0.8$ partially methylated, and $\beta<0.2$ unmethylated. An in-house methylation visualization app, MethylViz, developed using ShinyR, was used to visualize methylation values across chromosome 14 and at the regions of interest (code available upon request).

\section{Results}

\section{Clinical spectrum}

The male proband (BAB7004) is presently a 22-year-old. His clinical phenotype includes mild to moderate intellectual disability (ID), short stature $(<5 \%)$, apparent microcephaly $(<25 \%)$, and mild facial dysmorphisms. He was initially evaluated at 4 years of age due to short stature and speech delay, with a vocabulary of 10-20 single words. In retrospect, he was born at about 39 weeks gestation and weighed $2.6 \mathrm{~kg}$ ( $3 \mathrm{rd}$ centile). His growth and development were slow. He walked at 1.5 years. He had a broad nasal root, frontal bossing, anteverted nares, long eyelashes, high palate, micrognathia, mild pectus excavatum, brachydactyly with 2-3-4 partial toe syndactyly, hypermobility of joints, mild hypotonia, undescended testes, pes planus and small hands and feet. Blood chromosome analysis, echocardiogram and brain MRI were all normal. He returned for evaluation of mild to moderate ID and short stature. His physical exam at 22 years of age was similar to the findings listed above. However, he now had descended testes without surgical intervention. He now spoke in full sentences with articulation errors. Puberty had occurred at 16 years of age, and he was otherwise generally healthy. In addition to the proband, there are four healthy and developmentally normal siblings. The father is $178 \mathrm{~cm}$ and mother is $167.5 \mathrm{~cm}$, conferring a mid-parental height of about 179 $\mathrm{cm}$ for male offspring (63th centile for an adult male). Physical examination of the proband revealed height of $150 \mathrm{~cm}$ (-3.6 SD), weight $68 \mathrm{~kg}$ (50th centile) and occipital frontal circumference OFC $54 \mathrm{~cm}$ (25th centile).

\section{Whole genome sequencing}

A genomic DNA sample from individual BAB7004 was subjected to nanopore sequencing using two parallel libraries. The sheared DNA ONLL03750 library yielded a total of $51.7 \mathrm{Gbp}$ of genome sequence with 12,168,534 quality passed reads and a read N50 of $12,625 \mathrm{bp}$. About $64.14 \%$ of reads mapped to the hg38 haploid human reference genome. Of these, 224,165 reads mapped to chromosome 14 and 217,263 mapped to chromosome X. The mean coverage for this library of chr14 was $12.5 \mathrm{x}$ and for chrX was 5.9x. The full-length DNA ONLL03755 library yielded a total of 40.14 Gbp with $10,544,515$ quality passed reads with a read N50 of 10,504 bp. About $40.31 \%$ of the reads mapped to hg38 reference genome. Of those, 113,682 reads mapped to chromosome 14 and 108,428 mapped to chromosome $\mathrm{X}$. The mean coverage for the full-length ONLL03755 library of chr14 was 3.4x and for chrX was 1.6x. The mean coverage for the whole genome for ONLL03750 was 11.2x and for ONLL03755 was 3.0x.

\section{Characterization of triplication revealed a DUP-TRP/ INV-DUP structure}

A de novo copy-number gain spanning $\sim 2.2 \mathrm{Mb}$ consistent with heterozygous triplication (locus $\mathrm{CN}=4$ ) involving cytogenetic subbands 14q23.2q23.3 followed by $42.2 \mathrm{Mb}$ of copy-number neutral runs of homozygosity $(\mathrm{ROH}) /$ absence of heterozygosity $(\mathrm{AOH})$ spanning from 14q23.3q32.33 was identified in patient BAB7004 by a genome-wide custom-designed clinical chromosome microarray analysis (CMA) [27, 28](data not shown). Custom highresolution aCGH further revealed a complex genomic rearrangement (CGR) consisting of a large triplication $(2.2 \mathrm{Mb})$ flanked by small duplications at both centromeric $(26.2 \mathrm{~kb})$ and telomeric sides $(2.3 \mathrm{~kb})$ (Fig. 1). ES $\mathrm{CNV}$ calls were also analyzed along with the aCGH data to independently confirm the presence of a copynumber gain at chromosome 14 as well as to rule out any additional CNV that could have contributed to the genotype. ES analysis confirmed a copy-number gain call of $1.88 \mathrm{Mb}$ from chr14: 63,174,225-65,056,057 (hg19), which overlaps with the aCGH detected CNV of $2.2 \mathrm{Mb}$. No additional potential pathogenic CNVs were identified in the ES data by XHMM. Using BafCalculator [30, 31] on unphased ES data, and a comparison to genome-wide SNP array data, revealed a similar-sized $\mathrm{ROH} / \mathrm{AOH}$ copy-number neutral genomic interval mapping to chromosome 14.

Inverted triplications flanked by duplications is a CGR pattern referred to as DUP-TRP/INV-DUP [10] in which 
a

Patient: KCL204594

Coords: Chr14:62623366.6-65380206.4

Assembly: hg19
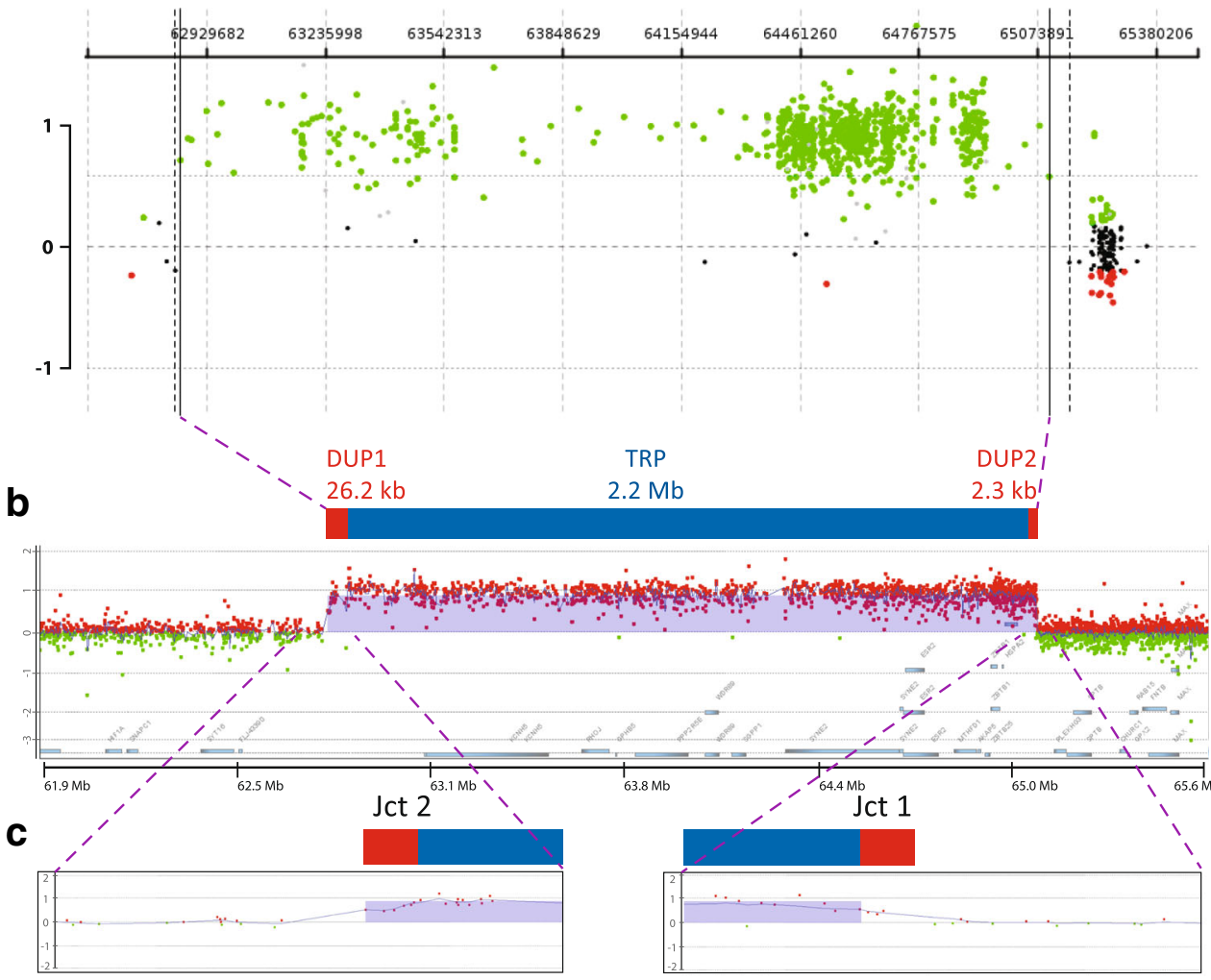

d

$62876674 \downarrow$

$65115460 \downarrow$

PROX (-) CAAGTCACAAAAAAATGGGGTTCACTGTAAACTGGTTTCAGTGTA PROX $(+)$ CAGGGGCCTCCACTGGCAGAAGAAAGAATCCCACAAGAGAC $\begin{array}{lll}\text { Jct2 CAAGTCACAAAAAAATGGGTTCAAAGGTCTATGAACAGCCAAGT } & \text { Jct1 CAGGGCCTCCACTGGCAGAAGCTCTGATTGGCTAGGCTTG } \\ \text { DIST (+) TGAGGAATTTGAGCCTGCATTTCAAAGGTCTATGAACAGCCAAGT } & \text { DIST (-) AGACGTAACTCCCAATAAAGAGCTCTGATTGGCTAGGCTTG }\end{array}$

$$
62850502 \uparrow \quad 65117723 \uparrow
$$

e

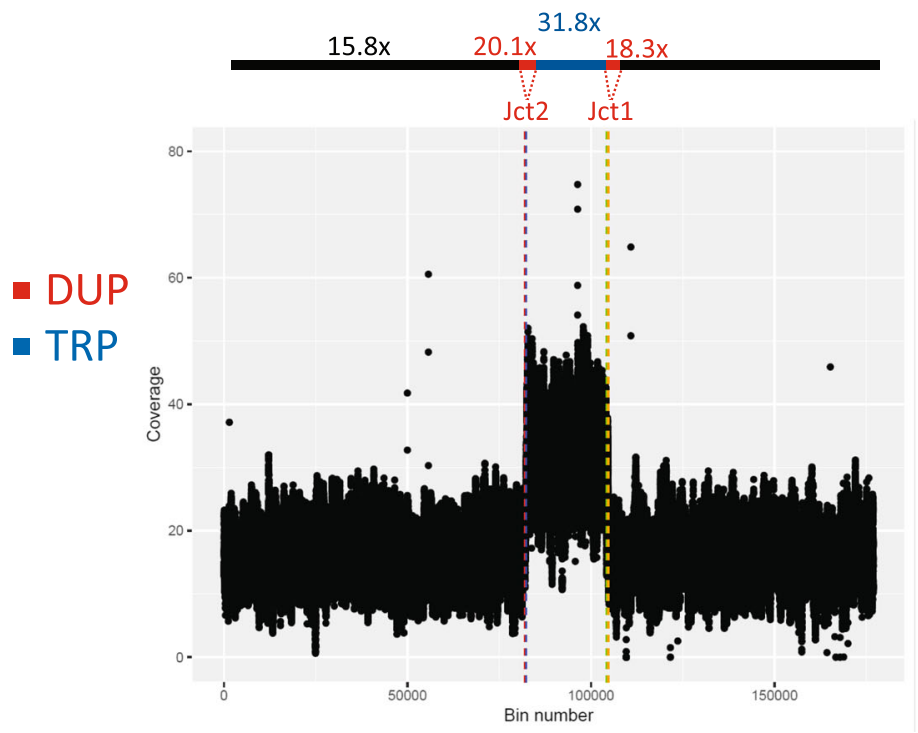

Fig. 1 (See legend on next page.) 
(See figure on previous page.)

Fig. 1 Triplication followed by ROH shows DUP-TRP/INV-DUP pattern. a Agilent aCGH (V10.1) plot of chromosome 14q23.2q23.3 region harboring a 2.2-Mb triplication (TRP; log2 ratio 1). b Agilent tiling high-density aCGH revealed a triplication (TRP; blue box) flanked by small duplications (DUP; red boxes) at both centromeric (Jct2) and telomeric junctions (Jct1) (c). d Color-matched sequence alignment of Jct1 and Jct2. Sequencing data of long-range PCR product was obtained by standard Sanger. Structure is now defined as DUP-TRP/INV-DUP. Genomic reference segments are displayed in blue (PROX, proximal) and red (DIST, distal). Strand orientation is indicated in parentheses. Microhomology at the junction is represented in black. e Read depth coverage plot of $17.7 \mathrm{Mb}$ segment of $14 \mathrm{q}$ (chr14: 54,179,300-71,874,900) that includes the DUP-TRP/INV-DUP region. Normalized read depth coverage confirms the relative copy-number differences along the CGR consistent with a triplication (31.8/15.8 $=2$ ) flanked by duplications (DUP1 $=20.1 / 15.8=1.3$; DUP2 $=18.3 / 15.8=1.2$ ). $Y$-axis: read depth coverage; $X$-axis: 100 bp bins at 14q. Genomic

coordinates are in hg19

the triplicated segment is inserted in an inverted orientation amid two duplicated copies with respect to the reference genome. We tested for this hypothesized CGR structure by designing PCR outward-oriented primers at apparent boundaries, as evidenced by change in copynumber states, of the copy-number gain segments (DUP-TRP or TRP-DUP), i.e., breakpoint junctions Jct1 and Jct2, as defined in Fig. 1. A single PCR band was obtained exclusively in the BAB7004 DNA sample; further Sanger sequencing confirmed that the triplicated segment is inverted, consistent with a DUP-TRP/INVDUP structure. Jct2 could not be obtained using this PCR junction amplification and Sanger sequencing approach, but it was further mapped by Nanopore long reads and validated by Sanger sequencing. Sequencing alignments are shown in Fig. 1d. There is a 2 bp and 4 bp of microhomology at Jct1 and Jct2, respectively; no additional complexity such as insertions, de novo single-nucleotide variants (SNVs) or indels were observed flanking $\sim 150 \mathrm{bp}$ of these sequence determined junctions [22, 47, 48].

Nanopore long-read sequencing, using algorithms that incorporate both split-reads and read depth data, supports the structure proposed for DUP-TRP/INV-DUP (Fig. 1e). Transition between copy-number neutral segments (15.8x) to flanking small duplications $(26.2 \mathrm{~kb}$ and $2.3 \mathrm{~kb})$ to the large triplication $(2.2 \mathrm{Mb})$ was possible using normalized read depth coverage values from $100 \mathrm{bp}$ bins calculated within the defined duplication junctions; Jct2 (chr14: 62,850,502-62,534,442) that defines flanking DUP1 (20.1x), Jct1 (chr14: 65,115,460 65,117,723) that defines DUP2 (18.3x), and (chr14: 62,876,674-65,115,460) that defines TRP (31.8x).

The relative normalized read depth coverage enables distinction among neutral copy number $(N=2)$, duplications (expected relative $\mathrm{CN}=1.5$; observed relative $\mathrm{CN}$ for DUP1 $=1.3$ and for DUP2 $=1.2$ ), and triplication (expected relative $\mathrm{CN}=2.0$; observed relative $\mathrm{CN}$ for $\mathrm{TRP}=2.0$ ) (Fig. 1e). The small size of flanking duplications (26 $\mathrm{kb}$ and $2.3 \mathrm{~kb}$ ) compared to the large size of the triplication $(2.2 \mathrm{Mb})$ likely contributes to both the absence of detection and resolution of the DUP1 and DUP2 by clinical CMA and the higher precision of the observed copy-number observation for long-read sequencing analysis for the TRP versus DUP.
Copy-number neutral ROH/AOH segment is due to perturbed transmission genetics and a segmental maternal uniparental disomy

The triplication gain was again confirmed by SNP array. $\mathrm{ROH} / \mathrm{AOH}$ spanning $42.2 \mathrm{Mb}$ of $14 \mathrm{q}$ that extends from the end of the triplicated segment until the telomere was identified in the diagnostic laboratory by CMA (Fig. 2a). We further confirmed the size of $\mathrm{ROH}$, equivalent to regions of $\mathrm{AOH}$, and genomic content by SNP array HumanOmniExpress-24 Beadchip (Fig. 2b) and ES analysis of $\mathrm{AOH}$ regions using BafCalculator. Both DUP-TRP/INV-DUP and AOH constitute a CGR that occurred de novo in individual BAB7004, and it is not present in his parents or unaffected siblings (Fig. $2 \mathrm{~b}$ and Additional file 1: Figure S1). SNP array revealed maternal inheritance of all SNPs in the AOH region indicating a segmental maternal isodisomy, and specifically UPD(14)mat. By calling SNVs on the nanopore generated data and calculating allele frequencies, the presence and extent of the $\mathrm{ROH} / \mathrm{AOH}$ could be independently corroborated.

\section{Triplication is formed by two segments with distinct genotypes}

Inspection of B-allele frequencies of the DUP-TRP/ INV-DUP segments revealed that the triplication was constituted by two distinct allele genotypes: a small, $\sim 92 \mathrm{~kb}$, region presenting SNPs with unequal parental allele contribution, i.e., ABBB or BAAA followed by a large segment of $\sim 2.2 \mathrm{Mb}$ with equal parental allele dosage, i.e., AABB (Fig. 2b, c). No copy-number differences exist between the two genotype states. Therefore, this result indicates that distinct parental chromosomal segments from homologous chromosomes were joined together without copy-number alteration. Such a transition site, arbitrarily named breakpoint junction 3 (Jct3), was previously observed in two out of five cases in our published cohort of triplications followed by $\mathrm{AOH}$ [22]. A model for the formation of this CGR in patient BAB7004 is shown in Fig. 2d. Flanking duplications of $26,2 \mathrm{~kb}$ and $2.3 \mathrm{~kb}$ were not detected by the high-resolution SNP array. 

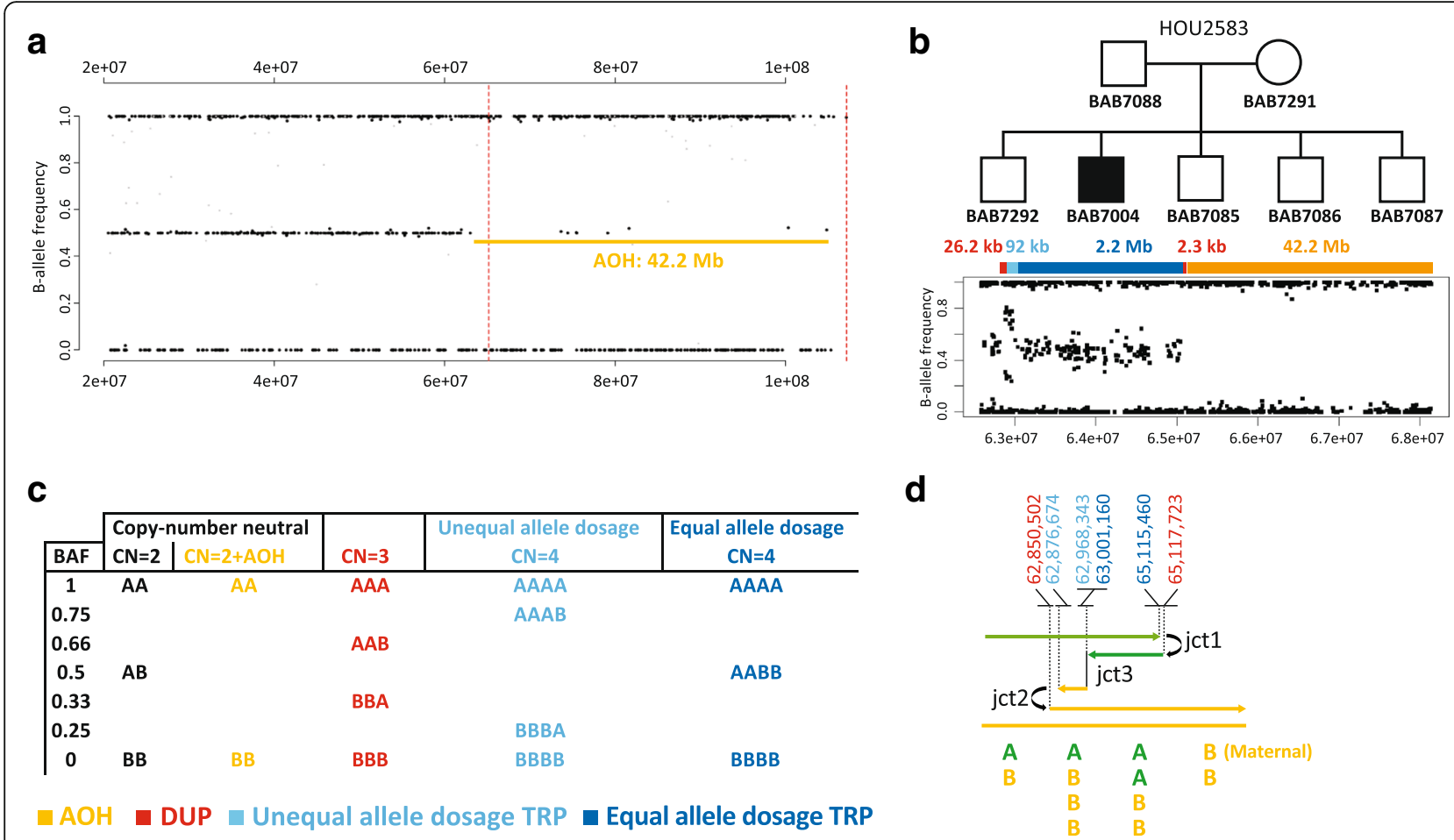

Fig. 2 SNP analysis enabled $\mathrm{AOH} / \mathrm{ROH}$ detection in addition to a copy-number neutral junction supporting underlying replication-based mechanism. a Agilent aCGH (V10.1) SNP array data (B-allele frequency, BAF) for chromosome 14 indicates a $42.2 \mathrm{Mb}$ of AOH. b Top: pedigree structure of family HOU2583; Bottom: Illumina SNP array HumanOmniExpress-24 Beadchip B-allele frequency (BAF) plot of $\sim 42 \mathrm{Mb}$ telomeric segment spanning 14q (chr14:62584057-107287663) reveals a de novo complex genomic rearrangement (CGR) and ROH/AOH exclusively present in BAB7004 (please refer to Additional file 1: Figure S1 for SNP array results in other family members). BAF revealed that the TRP segment presents two distinct genotypes: a small one $(92 \mathrm{~kb}$ ) with unequal allele dosage, followed by a larger one with equal allele dosage (2.2 Mb). Unequal allele dosage TRP, light blue rectangle; equal allele dosage TRP, dark blue rectangle; flanking small DUP, red rectangle; $\mathrm{AOH}$, orange rectangle. c Expected BAF genotypes for distinct copy-number states. $\mathbf{d}$ Color-matched schematic model of the 14q23.2q23.3 CGR formation in BAB7004. CGR presents at least three breakpoint junctions, i.e. Jct1, Jct2, Jct3, which are hypothesized to be generated by template switches during replication-based repair (see main text for discussion). Top: genomic coordinates of junctions inferred from multiple technical approaches. Bottom: representation of the SNP allele dosage in each segment involved in this CGR. A, B: SNP alleles. Genomic coordinates are in hg19

\section{Variants present in triplicated and copy-number neutral $\mathrm{ROH} / \mathrm{AOH}$ segments unlikely to contribute to the clinical phenotype}

We performed trio ES to access the presence of rare and/or potentially pathogenic variants in homozygosis within the $2.2-\mathrm{Mb}$ triplicated region and the $42.2-\mathrm{Mb}$ copy-number neutral $\mathrm{ROH} / \mathrm{AOH}$ genomic interval of chromosome 14 that may have potentially contributed to the clinical phenotype of patient BAB7004. Two rare missense variants affecting disease-associated genes, SYNE2 (NM_015180), chr14:g.64469886A>G (c.A4235G; p.E1412G) and POMT2 (NM_013382), and chr14:g.77744801A>T (c.T2083A; p.W695R) were detected and independently confirmed by Sanger sequencing. Heterozygous variants affecting SYNE2 cause autosomal dominant Emery-Dreifuss muscular dystrophy 5 (MIM\#612999). SYNE2 is within the triplicated segment; therefore, it is present in four copies. Since it maps to the equal dosage allele triplicated segment, reference and variant alleles are present two times each. The variant allele was inherited from the heterozygous mother, BAB7291 (Additional file 1: Figure S2a). In addition, this allele is not present in any of the following databases: our in-house database consisting of $\sim 9000$ exomes with a wide variety of Mendelian phenotypes; the Atherosclerosis Risk in Communities Study (ARIC) database of 11,000 exomes, dbSNP, or ExAC; and gnomAD databases. The variant affecting POMT2 was inherited from the heterozygous mother but it is present in the homozygous state in BAB7004 as it maps within the $\mathrm{AOH}$ region (Additional file 1: Figure S2b). However, although the subject presents with hypotonia, he does not have features of muscular dystrophy and does not have severe intellectual disability as can be observed in individuals with POMT2 pathogenic alleles. Therefore, in this clinical context, the homozygous variant is unlikely to contribute to his clinical phenotype.

Genome-wide analysis of de novo variants revealed a missense affecting the beta-tubulin class IIA gene, TUBB2A, (NM_001069), chr14: g. 3154999C>T (c.G436A, p.G146R). 
Importantly, part of $T U B B 2 A$ overlaps with four distinct segmental duplications which includes the exon on which such de novo variant maps. One of the segmental duplications consists of TUBB2B, which shares $>99 \%$ identical nucleotides and localizes $\sim 66 \mathrm{~kb}$ distal to TUBB2A in a direct orientation. To further validate the variant, we designed a primer pair to specifically amplify only the TUBB2A segment and not the other paralog sequences (Forward: 5' GCAAAACTGAGCACCATAG TT 3' and Reverse: 5' CCCAGTGTTTTTGAGGTCA CTG 3', amplicon size: 1327 bp). Standard Sanger sequencing confirmed the presence of the variant (Additional file 1: Figure S2c).

\section{Chromosome 14 methylation profile is consistent with maternal imprinting}

Chromosome 14 is subjected to imprinting; at least three differential methylation loci are known: IG-DMR and MEG3-DMR, both of which are hypermethylated in the paternal chromosome whereas hypomethylated in the maternal chromosome $[49,50]$ in addition to the recently described MEG8-DMR which is hypermethylated in the maternal chromosome [51] (Fig. 3a). Infinium Human Methylation450 Beadchip has probes mapping to the CpG sites of MEG3-DMR and MEG8-DMR both of which display methylation patterns consistent with maternal imprinting in BAB7004 (Fig. 3b). Finally, for the MEG3-DMR in BAB7004, we also used the nanopore sequencing reads for calling the base methylcytosine as one indication/readout of methylation status which confirmed the hypomethylation status of that locus compared to a non-UPD sample control (Fig. 3c).

\section{Discussion}

De novo TRP/AOH has been reported involving distinct chromosomes including $1,5,6,9,10,11,14$, and 22 [20-24, 52], in individuals presenting diverse phenotypes and congenital malformations. Here, we describe a family with a patient who carries a CGR, TRP/AOH, which is a rare combination of a CNV constituted by a $2.2 \mathrm{Mb}$ triplication plus a $42.2 \mathrm{Mb}$ segmental maternal isodisomy wherein the latter includes the imprinted locus at the $14 \mathrm{q} 32$ region. Each one of the patients carrying the TRP/AOH reported thus far has a distinct segment of the chromosome affected (for instance, 1p36.12, 6q24.1, 10q26.13, 14q32.12, 14q23.3q32.33, 9q12-q21.11) [22], therefore indicating that the underlying mechanism is not locus specific. Involvement of both parental chromosomes in the origin of triplicated segments indicates that such rearrangements are post-zygotic, likely occurring very early during development as no evidence of mosaicism was observed in the patients reported thus far $[20,21,23]$ potentially a perizygotic SV mutagenesis event [53]. In the subject reported here, analysis of both secondary somatically-imprinted DMR sites in 14q32, MEG3-DMR and MEG8-DMR, confirmed maternal specific imprinting patterns which supports the hypothesis that the TRP/ $\mathrm{AOH}$ occurred very early in development, likely before the blastocyst stage [50]. This unique family provides a compelling 'experiment of nature' in which one might dissect phenotypic consequence of trisomy 14 due to gene dosage effects (due to triplication or duplication) versus copy-number neutral chromosome 14 locus imprinting and transmission genetics reduction to homozygosity by $\mathrm{ROH} / \mathrm{AOH}$.

CGR breakpoint junction analysis by customized aCGH in the study subject, as well as patients published previously [22], revealed that the triplications are actually flanked by small duplications, the latter is often not detected by lower resolution clinical array CGH or CMA [22]. In addition, the triplications are inverted in all cases for which the orientation of the copy-number gain segments were studied [20, 21, 23, 24]. Molecular characterization unveiled that the currently studied family and patient ascertained TRP/AOH structure actually represents the same DUP-TRP/INV-DUP pattern described previously [10, 48] for triplications affecting diverse disease-associated loci genome-wide [54].

Here, we have used long-read single-molecule sequencing which independently confirms the DUP-TRP/INV-DUP structure previously proposed, i.e., an inverted triplication flanked by duplications, and enabled assessment of methylated bases at specific loci. The results of long-read genomic sequencing allowed read depth determinations to be used as a surrogate measure of relative copy number deriving an apparent "DUP-TRP-DUP" type of CGR structure as seen in a high-resolution array. Such a complex structure is formed by a microhomology-mediated break-induced replication (MMBIR) mechanism with at least two template switches that result in two breakpoint junctions, Jct1 and Jct2, corresponding to the insertion of the inverted triplication. Of note, a 2-bp and 4-bp microhomology were observed at Jct1 and Jct2, respectively, consistent with a microhomology -mediated template switch (TS). These "breakpoint junctions" can perhaps more accurately be thought of as "join-points" wherein a template switch, during MMBIR driven CGR formation, "joins" discontinuous sequence from the reference haploid genome together, creating an apparent breakpoint junction [22, 25, 54, 55]. Intriguingly, autosomal DUP-TRP/INV-DUP, as in the one reported here and others elsewhere [22], can be generated by TS between chromosome homologs, i.e., interchromosomal versus intrachromosomal recombination, in contrast to X-linked DUP-TRP/INV-DUP which are apparently preferentially generated by intrachromosomal TSs [10, 47]. Moreover, some of the autosomal DUP-TRP/INV-DUP are 


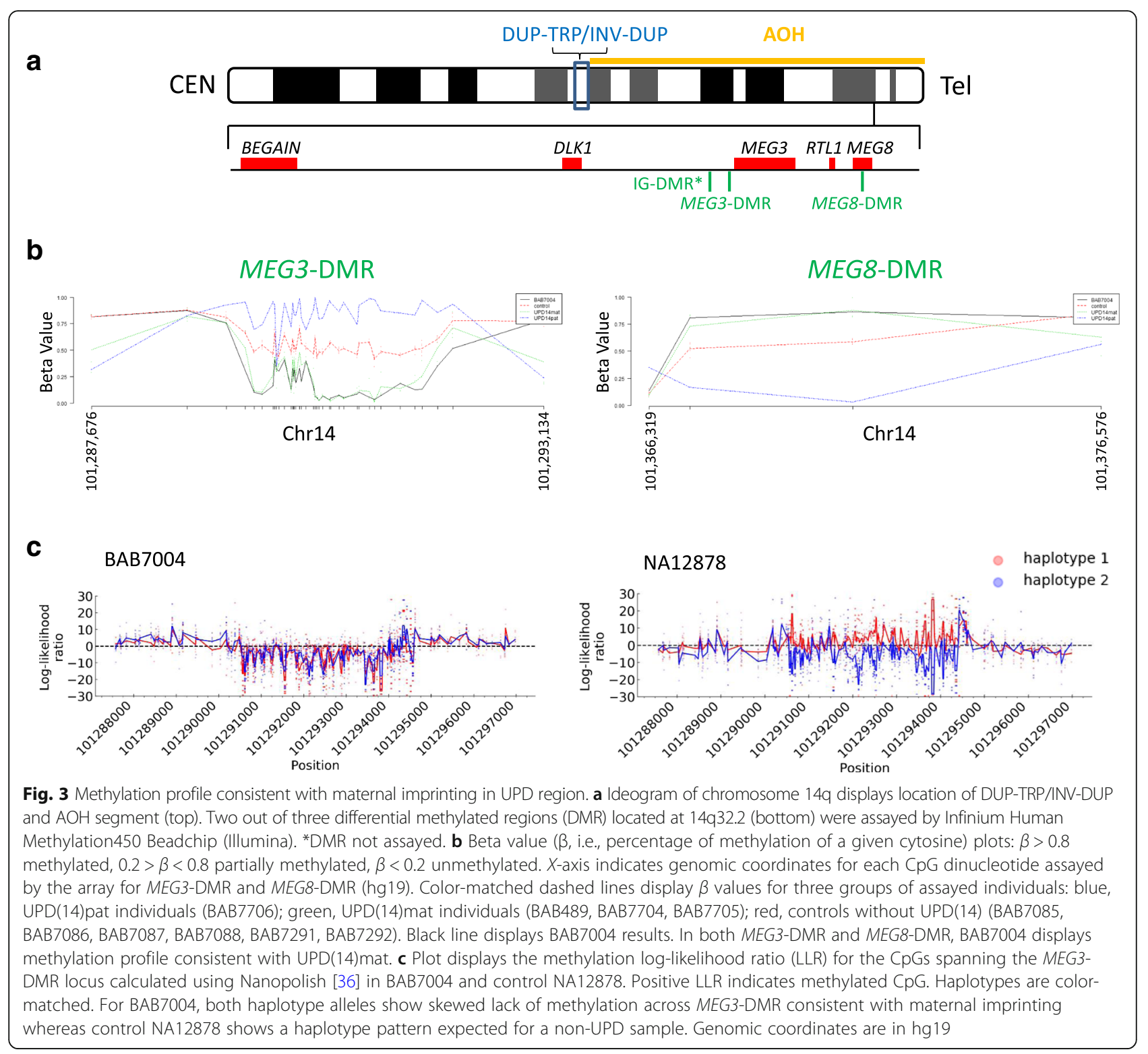

constituted by two distinct allele genotypes on which the transition between these genotypes constitutes a novel type of junction (Jct3) formed by template switches between homologous chromosomes without an accompanying copy-number alteration [22]. Such copy-number neutral junction, Jct3, also observed in this present CGR, has been hypothesized to be formed by template-switching between homologous chromosomes involving allelic rather than non-allelic segments, likely by a break-induced replication (BIR) mechanism of recombination [56], consistent with a copy-number neutral junction formation [22].

Each one of the three cases with evidence informative for a third template switch reported thus far involve a distinct chromosome, chromosome 14 in BAB7004 (described herein), chromosome 1 in DECIPHER_257814 and chromosome 10 in BAB3923 [22], but a similar pattern starts to emerge. For example: (i) all three cases are characterized as DUP-TRP/INV-DUP followed by AOH; (ii) the unequal parental allele contribution segment is small, varying in size from $126 \mathrm{~kb}$ to $150 \mathrm{~kb}$, whereas the equal parental allele contribution segments are large varying from $2.2 \mathrm{Mb}$ to $8.7 \mathrm{Mb}$; (iii) the unequal parental allele dosage segment maps close to Jct2 which is the transition junction from DUP-TRP/INV-DUP structure to $\mathrm{AOH}$ (Fig. 2d).

Maternal UPD(14) was first reported in the early 1990s: the syndrome as a clinical entity was reported by I.K.Temple and colleagues [57] for heterodisomy and by Liu et al. [26] for isodisomy. It is characterized by preand postnatal growth retardation, relative macrocephaly, hypotonia and motor delay, feeding problems early in 
life, truncal obesity, precocious puberty in both males and females, and facial characteristics including a broad forehead and fleshy nasal tip [58, 59]. Maternal UPD(14) syndrome presents overlapping clinical features with Prader-Willi (PWS, OMIM\#176270) [60] and Russel-Silver syndrome (SRS, OMIM\#180860) [61]. Molecular causes vary: a recent review of published cases culled from the literature [59] indicates that $\sim 78 \%$ of reported cases result from full isodisomy or heterodisomy UPD(14)mat, whereas paternal deletions on chromosome 14 spanning the 14q32 locus and loss of methylation at the intergenic DMR (IG-DMR) contribute to $\sim 10 \%$ and $\sim 12 \%$ of the molecular causes, respectively. Relevant for this study, segmental UPD14 has been very rarely reported in the literature [62]. Genotype-phenotype correlation in patients with a deletion affecting the paternal allele of chromosome 14 was important to delineate the imprinted genomic region [58]. In fact, Kagami and colleagues used such patients to narrow the region associated with maternal UPD(14) syndrome to $108 \mathrm{~kb}$ that includes imprinted genes DLK1 and GTL2/MEG3 as well as an important cis-regulatory DMR, IG-DMR [49].

In general, the patient presented here presents a clinical picture that overlaps significantly with the majority of subjects with UPD(14)mat [59], including short stature, small hands and feet, hypotonia, somewhat lax joints, and developmental delay; parents reported that he did not have early onset of puberty. Early puberty has been reported in $87 \%$ of patients with UPD(14)mat [59], $100 \%$ of patients carrying loss of methylation in the paternal germline IG-DMR at $14 \mathrm{q} 32$, and $2 / 3$ of the patients carrying deletion of the paternal $14 \mathrm{q} 32$. It should be noted, however, that some cases in the literature reported as "precocious" or "early" puberty do not meet standard clinical definitions and criteria for "precocious puberty". In contrast, this precocious puberty is mostly absent in partial maternal or paternal trisomy $14 \mathrm{q}$ [63], in deletions involving 14q32 from maternal origin [64], which indicates that early-onset puberty is triggered by the lack of expression of one of the paternally imprinted genes mapping within the 14q32 [59]. Consistent with this hypothesis, partial deletion of the paternally expressed gene $D L K 1$ was reported in a family with familial central precocious puberty when deletion was inherited from the father although this family shows incomplete penetrance for the trait [65]. Therefore, it is notable that the individual reported here does not have precocious puberty, although he has partial isodisomy UPD(14)mat which is associated with the expected patterns for this genomic finding (Fig. 3). One potential explanation is that the partial tetrasomy of $14 q 23.2 q 23.3$ compensates for the predicted lack of DLK1 expression or it changes the expression of the maternal copy which may preclude the phenotype of early-onset puberty. In addition a history of cryptorchidism is a known risk factor for pubertal delay.

The majority of subjects with UPD(14)mat have been reported to have normal intellectual development, although measured IQ, when available, has often been in the low normal range [59]. BAB7004 presents with intellectual disability and speech delay which may still be considered within the spectrum of what is generally reported in Temple syndrome [66]. The identified triplicated segment in subject 7004 spanning 14q23.2q23.3 does not have a known dosage-sensitive gene associated with ID/DD within its $2.2 \mathrm{Mb}$ interval. Partial trisomy of chromosome $14 \mathrm{q}$ is an extremely rare condition $[63,67]$, with extensive clinical variability which is consistent with the genomic content and size variability of the higher copy-number region involved in the cases reported [63].

The majority of individuals with trisomy 14 have craniofacial abnormalities, intellectual disabilities, neuromotor delay, and postnatal growth retardation. Very few individuals with trisomy 14 are reported with partial trisomy that overlaps the triplicated segment in the patient described here; however, we can not rule out a gene dosage effect either from direct alteration of the genomic copy number or disruption of regulatory regions, or topologically associated domains (TAD) for genes nearby the CNV. Of note, individuals with trisomy 14 mosaicism have a distinct and recognizable phenotype that shares little in common with the phenotype of UPD14 [68].

The phenotype of the UPD cases can also potentially be modulated or expanded by the contribution of expressed recessive disease traits. We investigated this possibility by performing trio ES analysis to search for homozygous pathogenic rare variants affecting known disease genes that map within the long-arm of chromosome 14. Within that segment, we found a rare homozygous missense mutation, c.T2083A:p.W695R, in POMT2 located within the maternally inherited UPD region. POMT2 is an endoplasmic reticulum membrane protein that, together with POMT1, catalyzes the first step in the synthesis of the $\mathrm{O}$-mannosyl glycan. This variant is present at a very low frequency in both dbSNP and ExAC $(\sim 0.015 \%)$ as well as gnomAD $(0.009 \%)$ and none of those variant databases report homozygous individuals. Pathogenic variants in POMT2 can cause one of three types of recessive muscular dystrophy-dystroglycanopathy: congenital with brain and eye anomalies, type A, 2 (MDDGA2, MIM\#613150); congenital with mental retardation, type B, 2 (MDDGB2, MIM\#613156); and limb-girdle, type C, 2 (MDDGC2, MIM\#613158). Although it is possible that the lower limb hypotonia and the intellectual disability found in this patient are caused by the biallelic variant allele in POMT2, this subject does not have features of muscular dystrophy; therefore, we consider this an unlikely possibility. 
In aggregate, these data provide experimental evidence that, in humans, triplication generated post-zygotically can lead to segmental UPD accompanied by an abnormal methylation pattern of specific loci mapping to chromosome 14. Furthermore, the impact on the disease phenotype resulting from the underlying replication-based mutational mechanism in humans can extend beyond that due to the formation of copy-number variants and recessive trait manifestations when only one parent is a carrier. In this study, we apodictically show traits associated with imprinted loci can manifest.

\section{Conclusions}

In summary, we report a 22-year-old male with segmental maternal uniparental isodisomy of chromosome 14 along with a DUP-TRP/INV-DUP that occurred post-zygotically by a template-switching replicative repair mechanism, most likely MMBIR. An apparent interchromosomal template switch results in methylation patterns of DMRs consistent with what is observed in individuals with UPD(14)mat. Therefore, intrachromosomal triplications generated by replication-based mechanisms can result in UPD and imprinting diseases. This case indicates that individuals with segmental UPD should have testing to specifically assess for CNVs that could have arisen postzygotically as part of the replicative process.

\section{Additional file}

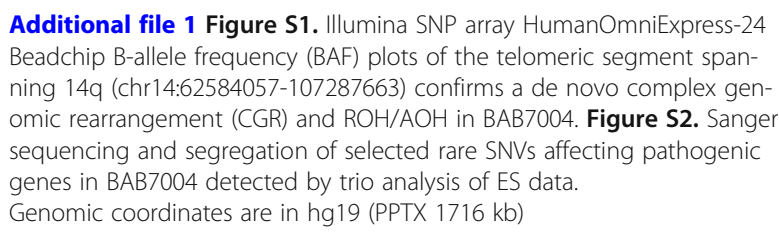

\section{Abbreviations}

aCGH: Array comparative genomic hybridization; $\mathrm{AOH}$ : Absence of heterozygosity; BIR: Break-induced replication; CADD: Combined annotationdependent depletion; CGRs: Complex genomic rearrangements; CNVs: Copynumber variants; DMRs: Differentially methylated regions; DUP-TRP/INVDUP: Inverted triplication flanked by duplications; FoSTeS: Fork stalling and template switching; MMBIR: Microhomology-mediated break-induced replication; NAHR: Nonallelic homologous recombination; OFC: Occipital frontal circumference; $\mathrm{ROH}$ : Runs of homozygosity; SNPs: Single-nucleotide polymorphisms; TRP: Triplications; UPD: Uniparental isodisomy

\section{Acknowledgements}

We thank the family and collaborators for their participation in this study. We also want to thank Jawid M. Fatih for his invaluable help with preparing the figures.

\section{Funding}

This work was funded in part by the US National Human Genome Research Institute (NHGRI)/ National Heart Lung and Blood Institute (NHLBI) grant number UM1HG006542 to the Baylor-Hopkins Center for Mendelian Genomics (BHCMG), the National Institute of Neurological Disorders and Stroke (NINDS) R35 NS105078 (J.R.L.), and the Eunice Kennedy Shriver National Institute of Child Health \& Human Development (NICHD) 1R03HD092569-01A1 (C.M.B.C.). The project was supported in part by IDDRC grant number 1 U54 HD083092 from the NICHD.

\section{Availability of data and materials}

The datasets of trio whole-exome sequencing DNA sequences generated during the current study are available in the database of Genotypes and Phenotypes (dbGaP) (https://www.ncbi.nlm.nih.gov/gap). The accession number is phs00071 1.v5.p1, under the Baylor Hopkins Center for Mendelian Genomics study. Sample identifiers are SRS2853004, SRS2796466, and SRS2773868.

\section{Authors' contributions}

CMBC participated in the design of the study, performed the majority of the analyses/experiments, and prepared the manuscript with JRL. NAH, RSK, JWB, and JB participated in the methylation study and performed analyses. BY performed aCGH experiments, BY and $\mathrm{HH}$ performed Sanger sequencing CGR breakpoint junction validation, and HH performed validation of gene candidate variants. ZCA and CAS contributed to the statistical and bioinformatic analyses of the genomics and phenotype data. PS, SWC, VRS, and PIB participated in the interpretation of clinical phenotype and molecular evidence as well as critically revised the manuscript. DJT and SJ participated in the design of the nanopore sequencing component of the study, and JB, MP, and EH conducted the analysis of long-read sequencing data. SNJ, HD, and RAG participated in the design of the study, generated the ES data, and critically revised the manuscript. EB and RAG participated in the design of the study and the overview of ES informatics pipeline and critically revised the manuscript. JRL participated in the design of the study, analyses of all molecular and clinical data, and data interpretation; oversaw the manuscript preparation; and critically revised the manuscript. All authors approved the final submitted version of the manuscript and are accountable for the accuracy and integrity of the work.

\section{Ethics approval and consent to participate}

Informed consent was obtained for participation in the research study. This study was approved by the Baylor Institutional Review Board (IRB) at Baylor College of Medicine (protocols H-25466 and/or H-29697). This study was performed within the guidelines of the Declaration of Helsinki.

\section{Consent for publication}

A written consent was obtained to publish clinical information of the patient reported here.

\section{Competing interests}

Baylor College of Medicine (BCM) and Miraca Holdings have formed a joint venture with shared ownership and governance of the Baylor Genetics (BG), which performs clinical microarray analysis and clinical exome sequencing. C.A.S. is an employee of BCM and derives support through a professional services agreement with the BG. J.R.L. serves on the Scientific Advisory Board of the BG. J.R.L. has stock ownership in 23andMe, is a paid consultant for Regeneron Pharmaceuticals, and is a co-inventor on multiple United States and European patents related to molecular diagnostics for inherited neuropathies, eye diseases, and bacterial genomic fingerprinting. The Department of Molecular and Human Genetics at Baylor College of Medicine derives revenue from molecular genetic testing offered in the Baylor Genetics Laboratories. J.W.B. is currently a full-time employee of Illumina, Inc. though all contributions to this work took place while he was an employee of BCM. MP, E.H., J.B., S.J., and D.J.T. are full-time employees of, and stock option holders in, Oxford Nanopore Technologies. The remaining authors declare that they have no competing interests.

\section{Publisher's Note}

Springer Nature remains neutral with regard to jurisdictional claims in published maps and institutional affiliations.

\section{Author details}

${ }^{1}$ Department of Molecular and Human Genetics, Baylor College of Medicine, One Baylor Plaza, Room 604B, Houston, TX 77030-3498, USA. ${ }^{2}$ Oxford Nanopore Technologies Inc, New York, NY, USA. ${ }^{3}$ Oxford Nanopore Technologies Inc, San Francisco, CA, USA. ${ }^{4}$ Oxford Nanopore Technologies Ltd, Oxford, UK. ${ }^{5}$ UT MD Anderson Cancer Center, Houston, TX, USA. ${ }^{6}$ Human Genome Sequencing Center, Baylor College of Medicine, Houston, TX 77030, USA. ${ }^{7}$ Department of Pediatrics, Baylor College of Medicine, Houston, TX 77030, USA. ${ }^{8}$ Texas Children's Hospital, Houston, TX, USA. ${ }^{9}$ Carle Clinic, Urbana, IL 61801, USA. 
Received: 12 December 2018 Accepted: 2 April 2019 Published online: 23 April 2019

\section{References}

1. Riccardi VM, Lupski JR. Duplications, deletions, and single-nucleotide variations: the complexity of genetic arithmetic. Genet Med. 2013;15(3):172-3.

2. Wolf NI, Sistermans EA, Cundall M, Hobson GM, Davis-Williams AP, Palmer R, et al. Three or more copies of the proteolipid protein gene PLP1 cause severe Pelizaeus-Merzbacher disease. Brain. 2005;128(Pt 4):743-51.

3. del Gaudio D, Fang P, Scaglia F, Ward PA, Craigen WJ, Glaze DG, et al. Increased MECP2 gene copy number as the result of genomic duplication in neurodevelopmentally delayed males. Genet Med. 2006;8(12):784-92.

4. Ishmukhametova A, Chen JM, Bernard R, de Massy B, Baudat F, Boyer A, et al. Dissecting the structure and mechanism of a complex duplicationtriplication rearrangement in the DMD gene. Hum Mutat. 2013;34(8):1080-4.

5. Lupianez DG, Kraft K, Heinrich V, Krawitz P, Brancati F, Klopocki E, et al. Disruptions of topological chromatin domains cause pathogenic rewiring of gene-enhancer interactions. Cell. 2015;161(5):1012-25.

6. Franke M, Ibrahim DM, Andrey G, Schwarzer W, Heinrich V, Schopflin R, et al. Formation of new chromatin domains determines pathogenicity of genomic duplications. Nature. 2016;538(7624):265-9.

7. Zuccherato LW, Alleva B, Whiters MA, Carvalho CM, Lupski JR. Chimeric transcripts resulting from complex duplications in chromosome Xq28. Hum Genet. 2016;135(2):253-6.

8. Wang JC, Fisker T, Dang L, Teshima I, Nowaczyk MJ. 4.3-Mb triplication of 4q32.1-q32.2: report of a family through two generations. Am J Med Genet A. 2009;149A(10):2274-9.

9. Beunders $G$, van de Kamp JM, Veenhoven $R H$, van Hagen JM, Nieuwint AW, Sistermans EA. A triplication of the Williams-Beuren syndrome region in a patient with mental retardation, a severe expressive language delay, behavioural problems and dysmorphisms. J Med Genet. 2010;47(4):271-5.

10. Carvalho CM, Ramocki MB, Pehlivan D, Franco LM, Gonzaga-Jauregui C, Fang $P$, et al. Inverted genomic segments and complex triplication rearrangements are mediated by inverted repeats in the human genome. Nat Genet. 2011:43(11):1074-81.

11. Liu P, Gelowani V, Zhang F, Drory VE, Ben-Shachar S, Roney E, et al. Mechanism, prevalence, and more severe neuropathy phenotype of the Charcot-Marie-Tooth type 1A triplication. Am J Hum Genet. 2014;94(3): 462-9.

12. Farrer M, Kachergus J, Forno L, Lincoln S, Wang DS, Hulihan M, et al. Comparison of kindreds with parkinsonism and alpha-synuclein genomic multiplications. Ann Neurol. 2004;55(2):174-9.

13. Fuchs J, Nilsson C, Kachergus J, Munz M, Larsson EM, Schule B, et al. Phenotypic variation in a large Swedish pedigree due to SNCA duplication and triplication. Neurology. 2007;68(12):916-22.

14. Chauvin A, Chen JM, Quemener S, Masson E, Kehrer-Sawatzki H, Ohmle B, et al. Elucidation of the complex structure and origin of the human trypsinogen locus triplication. Hum Mol Genet. 2009;18(19):3605-14.

15. Van Dijck A, van der Werf IM, Reyniers E, Scheers S, Azage M, Siefkas K, et al. Five patients with a chromosome 1q21.1 triplication show macrocephaly, increased weight and facial similarities. Eur J Med Genet. 2015;58(10):503-8.

16. Lopez-Exposito I, Bafalliu JA, Santos M, Fuster C, Puche-Mira A, GuillenNavarro E. Intrachromosomal partial triplication of chromosome 13 secondary to a paternal duplication with mild phenotypic effect. Am J Med Genet A. 2008;146A(9):1190-4.

17. Liu P, Erez A, Nagamani SC, Bi W, Carvalho CM, Simmons AD, et al. Copy number gain at Xp22.31 includes complex duplication rearrangements and recurrent triplications. Hum Mol Genet. 2011;20(10):1975-88.

18. Gu S, Posey JE, Yuan B, Carvalho CM, Luk HM, Erikson K, et al. Mechanisms for the generation of two quadruplications associated with split-hand malformation. Hum Mutat. 2016;37(2):160-4

19. Reddy KS, Logan JJ. Intrachromosomal triplications: molecular cytogenetic and clinical studies. Clin Genet. 2000;58(2):134-41.

20. Beneteau C, Landais E, Doco-Fenzy M, Gavazzi C, Philippe C, Beri-Dexheimer $M$, et al. Microtriplication of 11q24.1: a highly recognisable phenotype with short stature, distinctive facial features, keratoconus, overweight, and intellectual disability. J Med Genet. 2011:48(9):635-9.

21. Fujita A, Suzumura H, Nakashima M, Tsurusaki Y, Saitsu H, Harada N, et al. A unique case of de novo 5q33.3-q34 triplication with uniparental isodisomy of 5q34-qter. Am J Med Genet A. 2013;161A(8):1904-9.
22. Carvalho CM, Pfundt R, King DA, Lindsay SJ, Zuccherato LW, Macville MV, et al. Absence of heterozygosity due to template switching during replicative rearrangements. Am J Hum Genet. 2015;96(4):555-64.

23. Sahoo T, Wang JC, Elnaggar MM, Sanchez-Lara P, Ross LP, Mahon LW, et al. Concurrent triplication and uniparental isodisomy: evidence for microhomology-mediated break-induced replication model for genomic rearrangements. Eur J Hum Genet. 2015;23(1):61-6.

24. Xiao B, Xu H, Ye H, Hu Q, Chen Y, Qiu W. De novo 11q13.4q14.3 tetrasomy with uniparental isodisomy for 11q14.3qter. Am J Med Genet A. 2015; 167A(10):2327-33.

25. Hastings PJ, Ira G, Lupski JR. A microhomology-mediated break-induced replication model for the origin of human copy number variation. PLOS Genet. 2009a;5(1):e1000327.

26. Pentao L, Lewis RA, Ledbetter DH, Patel PI, Lupski JR. Maternal uniparental isodisomy of chromosome 14: association with autosomal recessive rod monochromacy. Am J Hum Genet. 1992;50(4):690-9.

27. Cheung SW, Shaw CA, Yu W, Li J, Ou Z, Patel A, et al. Development and validation of a CGH microarray for clinical cytogenetic diagnosis. Genet Med. 2005;7(6):422-32.

28. Wiszniewska J, Bi W, Shaw C, Stankiewicz P, Kang SH, Pursley AN, et al. Combined array CGH plus SNP genome analyses in a single assay for optimized clinical testing. Eur J Hum Genet. 2014;22(1):79-87.

29. Sutton VR, McAlister WH, Bertin TK, Kaffe S, Wang JC, Yano S, et al. Skeletal defects in paternal uniparental disomy for chromosome 14 are recapitulated in the mouse model (paternal uniparental disomy 12). Hum Genet. 2003;113(5):447-51.

30. Gambin T, Akdemir ZC, Yuan B, Gu S, Chiang T, Carvalho CMB, et al. Homozygous and hemizygous CNV detection from exome sequencing data in a Mendelian disease cohort. Nucleic Acids Res. 2017:45(4):1633-48.

31. Karaca E, Posey JE, Coban Akdemir Z, Pehlivan D, Harel T, Jhangiani SN, et al. Phenotypic expansion illuminates multilocus pathogenic variation. Genet Med. 2018;20(12);1528-37.

32. Sedlazeck FJ, Rescheneder P, Smolka M, Fang H, Nattestad M, von Haeseler $A$, et al. Accurate detection of complex structural variations using singlemolecule sequencing. Nat Methods. 2018;15(6):461-8.

33. Thorvaldsdottir H, Robinson JT, Mesirov JP. Integrative Genomics Viewer (IGV): high-performance genomics data visualization and exploration. Brief Bioinform. 2013;14(2):178-92.

34. Pedersen BS, Quinlan AR. Mosdepth: quick coverage calculation for genomes and exomes. Bioinformatics. 2018;34(5):867-8.

35. Li H. Minimap2: pairwise alignment for nucleotide sequences. Bioinformatics. 2018;34(18):3094-100.

36. Simpson JT, Workman RE, Zuzarte PC, David M, Dursi LJ, Timp W. Detecting DNA cytosine methylation using nanopore sequencing. Nat Methods. 2017; 14(4):407-10.

37. Bainbridge MN, Wang M, Wu Y, Newsham I, Muzny DM, Jefferies JL, et al. Targeted enrichment beyond the consensus coding DNA sequence exome reveals exons with higher variant densities. Genome Biol. 2011;12(7):R68.

38. Challis D, Yu J, Evani US, Jackson AR, Paithankar S, Coarfa C, et al. An integrative variant analysis suite for whole exome next-generation sequencing data. BMC Bioinformatics. 2012;13:8.

39. Reid JG, Carroll A, Veeraraghavan N, Dahdouli M, Sundquist A, English A, et al. Launching genomics into the cloud: deployment of Mercury, a next generation sequence analysis pipeline. BMC Bioinformatics. 2014;15:30.

40. Eldomery MK, Coban-Akdemir Z, Harel T, Rosenfeld JA, Gambin T, StrayPedersen $A$, et al. Lessons learned from additional research analyses of unsolved clinical exome cases. Genome Med. 2017;9(1):26.

41. Adzhubei IA, Schmidt S, Peshkin L, Ramensky VE, Gerasimova A, Bork P, et al. A method and server for predicting damaging missense mutations. Nat Methods. 2010;7(4):248-9.

42. $\operatorname{Sim} \mathrm{NL}$, Kumar $\mathrm{P}, \mathrm{Hu}$ J, Henikoff S, Schneider G, Ng PC. SIFT web server: predicting effects of amino acid substitutions on proteins. Nucleic Acids Res. 2012:40(Web Server issue:W452-7.

43. Chun S, Fay JC. Identification of deleterious mutations within three human genomes. Genome Res. 2009;19(9):1553-61.

44. Schwarz JM, Rodelsperger C, Schuelke M, Seelow D. MutationTaster evaluates disease-causing potential of sequence alterations. Nat Methods. 2010;7(8):575-6.

45. Kircher M, Witten DM, Jain P, O'Roak BJ, Cooper GM, Shendure J. A general framework for estimating the relative pathogenicity of human genetic variants. Nat Genet. 2014:46(3):310-5. 
46. Fromer M, Moran JL, Chambert K, Banks E, Bergen SE, Ruderfer DM, et al. Discovery and statistical genotyping of copy-number variation from whole-exome sequencing depth. Am J Hum Genet. 2012;91(4):597-607.

47. Carvalho CM, Pehlivan D, Ramocki MB, Fang P, Alleva B, Franco LM, et al. Replicative mechanisms for CNV formation are error prone. Nat Genet. 2013; 45(11):1319-26.

48. Beck CR, Carvalho CM, Banser L, Gambin T, Stubbolo D, Yuan B, et al. Complex genomic rearrangements at the PLP1 locus include triplication and quadruplication. PLoS Genet. 2015;11(3):e1005050.

49. Kagami M, Sekita Y, Nishimura G, Irie M, Kato F, Okada M, et al. Deletions and epimutations affecting the human $14 q 32.2$ imprinted region in individuals with paternal and maternal upd(14)-like phenotypes. Nat Genet. 2008;40(2):237-42.

50. Beygo J, Kuchler A, Gillessen-Kaesbach G, Albrecht B, Eckle J, Eggermann T, et al. New insights into the imprinted MEG8-DMR in 14q32 and clinical and molecular description of novel patients with Temple syndrome. Eur J Hum Genet. 2017;25(8):935-45.

51. Court F, Tayama C, Romanelli V, Martin-Trujillo A, Iglesias-Platas I, Okamura K, et al. Genome-wide parent-of-origin DNA methylation analysis reveals the intricacies of human imprinting and suggests a germline methylation-independent mechanism of establishment. Genome Res. 2014;24(4):554-69.

52. Kekis M, Deeg C, Hashimoto S, McKinney A, Erdman L, Green-Geer C, et al. Partial tetrasomy $11 \mathrm{q}$ resulting from an intrachromosomal triplication of a $22 \mathrm{Mb}$ region of chromosome 11. Am J Med Genet A. 2017;173(4):1056-60.

53. Liu P, Yuan B, Carvalho CMB, Wuster A, Walter K, Zhang L, et al. An organismal CNV mutator phenotype restricted to early human development. Cell. 2017;168(5):830-842 e837.

54. Carvalho CM, Lupski JR. Mechanisms underlying structural variant formation in genomic disorders. Nat Rev Genet. 2016;17(4):224-38.

55. Lee JA, Carvalho CM, Lupski JR. A DNA replication mechanism for generating nonrecurrent rearrangements associated with genomic disorders. Cell. 2007;131(7):1235-47.

56. Smith CE, Llorente $B$, Symington LS. Template switching during breakinduced replication. Nature. 2007:447(7140):102-5.

57. Temple IK, Cockwell A, Hassold T, Pettay D, Jacobs P. Maternal uniparental disomy for chromosome 14. J Med Genet. 1991;28(8):511-4.

58. Sutton VR, Shaffer LG. Search for imprinted regions on chromosome 14: comparison of maternal and paternal UPD cases with cases of chromosome 14 deletion. Am J Med Genet. 2000;93(5):381-7.

59. Ioannides Y, Lokulo-Sodipe K, Mackay DJ, Davies JH, Temple IK. Temple syndrome: improving the recognition of an underdiagnosed chromosome 14 imprinting disorder: an analysis of 51 published cases. J Med Genet. 2014;51(8):495-501.

60. Mitter D, Buiting K, von Eggeling F, Kuechler A, Liehr T, Mau-Holzmann UA, et al. Is there a higher incidence of maternal uniparental disomy 14 [upd(14)mat]? Detection of 10 new patients by methylation-specific PCR. Am J Med Genet A. 2006;140(19):2039-49.

61. Poole RL, Docherty LE, Al Sayegh A, Caliebe A, Turner C, Baple E, et al. Targeted methylation testing of a patient cohort broadens the epigenetic and clinical description of imprinting disorders. Am J Med Genet A. 2013; $161 \mathrm{~A}(9): 2174-82$.

62. Gillessen-Kaesbach G, Albrecht B, Eggermann T, Elbracht M, Mitter D, Morlot $\mathrm{S}$, et al. Molecular and clinical studies in 8 patients with Temple syndrome. Clin Genet. 2018;93(6):1179-88.

63. Bregand-White J, Saller DN, Clemens M, Surti U, Yatsenko SA, Rajkovic A. Genotype-phenotype correlation and pregnancy outcomes of partial trisomy 14q: a systematic review. Am J Med Genet A. 2016;170(9):2365-71.

64. Rosenfeld JA, Fox JE, Descartes M, Brewer F, Stroud T, Gorski JL, et al. Clinical features associated with copy number variations of the $14 q 32$ imprinted gene cluster. Am J Med Genet A. 2015;167A(2):345-53.

65. Dauber A, Cunha-Silva M, Macedo DB, Brito VN, Abreu AP, Roberts SA, et al. Paternally inherited DLK1 deletion associated with familial central precocious puberty. J Clin Endocrinol Metab. 2017;102(5):1557-67.

66. Briggs TA, Lokulo-Sodipe K, Chandler KE, Mackay DJ, Temple IK. Temple syndrome as a result of isolated hypomethylation of the $14 \mathrm{q} 32$ imprinted DLK1/MEG3 region. Am J Med Genet A. 2016;170A(1):170-5.
67. Brewer C, Holloway S, Zawalnyski P, Schinzel A, FitzPatrick D. A chromosomal duplication map of malformations: regions of suspected haplo- and triplolethality--and tolerance of segmental aneuploidy--in humans. Am J Hum Genet. 1999:64(6):1702-8.

68. Shinawi M, Shao L, Jeng $\sqcup$, Shaw CA, Patel A, Bacino C, et al. Low-level mosaicism of trisomy 14: phenotypic and molecular characterization. Am J Med Genet A. 2008;146A(11):1395-405.
Ready to submit your research? Choose BMC and benefit from:

- fast, convenient online submission

- thorough peer review by experienced researchers in your field

- rapid publication on acceptance

- support for research data, including large and complex data types

- gold Open Access which fosters wider collaboration and increased citations

- maximum visibility for your research: over $100 \mathrm{M}$ website views per year

At BMC, research is always in progress.

Learn more biomedcentral.com/submissions 